\title{
Towards the black hole uniqueness: transverse deformations of the extremal Reissner-Nordström-(A)dS horizon
}

\section{Maciej Kolanowski}

Institute of Theoretical Physics, Faculty of Physics, University of Warsaw, Pasteura 5, 02-093 Warsaw, Poland

E-mail: Maciej.Kolanowski@fuw.edu.pl

ABSTRACT: We study all transverse deformations of the extremal Reissner-Nordström(A)dS horizon in the Einstein-Maxwell theory. No symmetry assumptions are needed. It is shown, that for the generic values of a charge, the only allowed deformation is spherically symmetric. However, it is shown that for fine-tuned values of the charge, the space of deformations is larger, yet still finite-dimensional.

KEYwords: Black Holes, Classical Theories of Gravity

ArXiv EPRINT: 2111.00806 


\section{Contents}

1 Introduction $\quad 1$

1.1 Near horizon geometry limit 2

1.2 Deformations 3

2 Classification of the transverse deformations 3

2.1 Background 3

2.2 Deformations 4

2.3 Solutions 6

3 Discussion $\quad 7$

$\begin{array}{lll}3.1 & \text { Physical interpretation } & 7\end{array}$

$\begin{array}{lll}3.2 & \text { Connection to the previous work } & 8\end{array}$

$\begin{array}{ll}3.3 \text { Generalizations and further work } & 9\end{array}$

$\begin{array}{llr}\text { 3.3.1 Higher dimensional horizons } & 9\end{array}$

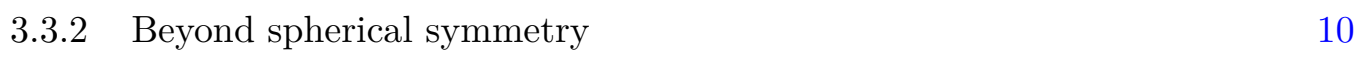

$\begin{array}{ll}\text { A Technical details } & 10\end{array}$

\section{Introduction}

The idea of the black hole uniqueness started with the seminal work of Israel [1]. The topic was heavily investigated for over fifty years now with a notable results from Hawking [2], Carter [3], Robinson [4], Mazur [5], Chruściel and Wald [6]. For the modern review, see [7].

Killing horizons, which are central objects in virtually all uniqueness theorems, can be divided into two categories: non-degenerate or degenerate (extremal) ones, on which Killing vector fields are affinely parametrized. This second type required separate analysis, in particular solving the infamous Near Horizon Geometry equation (1.5). In this paper we will focus entirely on the degenerate case, albeit from a different perspective: given a solution to (1.5), in what ways can we extend it to the bulk?

The question of the black holes' uniqueness changes drastically when one introduces a cosmological constant. Indeed, there is a plethora of static solutions to the EinsteinMaxwell- $\Lambda$ equations which are asymptotically AdS, and they do not possess any spatial symmetry [8]. On the other hand, it may be shown that the only static NHG with the electromagnetic field is given by the extremal Reissner-Nordström-(A)dS [9], thus suggesting that the extremal case is more rigid when $\Lambda \neq 0$. We will further strengthen this result, showing that the first-order deformations must be spherically-symmetric as well. 


\subsection{Near horizon geometry limit}

On the neighborhood of the extremal horizon, one can introduce a null gaussian coordinate system in which the metric reads:

$$
g=2 d v\left(d r+r h_{a} d x^{a}+\frac{1}{2} r^{2} F d v\right)+\gamma_{a b} d x^{a} d x^{b},
$$

where the horizon is located at $r=0, x^{a}$ are coordinates on the cross-section (a sphere, in our case) and $F, h_{a}, \gamma_{a b}$ depend upon $r$ and $x^{a}$ only. The Killing vector field is given by $\partial_{v}$. Maxwell field around $r=0$ admits a similar form: ${ }^{1}$

$$
\mathcal{F}=\Psi d v \wedge d r+r W_{a} d v \wedge d x^{a}+Z_{a} d r \wedge d x^{a}+\frac{1}{2} B_{a b} d x^{a} \wedge d x^{b},
$$

where $\Psi, W_{a}, Z_{a}, B_{a b}$ are smooth and depend only upon $r, x^{a}$.

We may now consider a one-parameter family of diffeomorphisms

$$
\phi_{\epsilon}\left(v, r, x^{a}\right)=\left(\epsilon^{-1} v, \epsilon r, x^{a}\right)
$$

for $\epsilon>0$. Quite remarkably, objects $g(\epsilon):=\phi_{\epsilon}^{\star} g$ and $\mathcal{F}(\epsilon):=\phi_{\epsilon}^{\star} \mathcal{F}$ possess well-defined limit when $\epsilon \rightarrow 0$. Since $\phi_{\epsilon}$ is no longer diffeomorphism in this limit, it may be an entirely new (yet much simpler) solution to the Einstein-Maxwell equations. Indeed, we can write those limits as

$$
\begin{aligned}
\lim _{\epsilon \rightarrow 0} \phi_{\epsilon}^{\star} g & =2 d v\left(d r+r h_{a}^{(0)} d x^{a}+\frac{1}{2} r^{2} F^{(0)} d v\right)+\gamma_{a b}^{(0)} d x^{a} d x^{b} \\
\lim _{\epsilon \rightarrow 0} \phi_{\epsilon}^{\star} \mathcal{F} & =\Psi^{(0)} d v \wedge d r+r W_{a}^{(0)} d v \wedge d x^{a}+\frac{1}{2} B_{a b}^{(0)} d x^{a} \wedge d x^{b},
\end{aligned}
$$

where all objects $h_{a}^{(0)}, F^{(0)}, \Psi^{(0)}, W_{a}^{(0)}, B_{a b}^{(0)}$ are now $r$ independent, and they are given by the limits as $r \rightarrow 0$ of associated objects in the original spacetime. Einstein-Maxwell equations in this setting read

$$
\begin{aligned}
R_{a b}^{(0)} & =\frac{1}{2} h_{a}^{(0)} h_{b}^{(0)}-D_{(a} h_{b)}^{(0)}+\Lambda \gamma_{a b}^{(0)}+2 B_{a c} B_{b}^{(0) c}+\frac{2}{D-2} \gamma_{a b}^{(0)} \Psi^{(0) 2}-\frac{1}{D-2} \gamma_{a b}^{(0)} B^{(0) 2} \\
F^{(0)} & =\frac{1}{2} h^{(0) 2}-\frac{1}{2} D^{a} h_{a}^{(0)}+\Lambda-2 \frac{D-3}{D-2} \Psi^{(0) 2}-\frac{1}{D-2} B^{(0) 2} \\
W^{(0)} & =d \Psi^{(0)} \\
d B^{(0)} & =0 \\
\left(D_{a}\right. & \left.-h_{a}^{(0)}\right) \Psi^{(0)}+\left(D^{b}-h^{b(0)}\right) B_{b a}=0
\end{aligned}
$$

where $D$ is the number of spacetime dimensions, $D_{a}$ is the covariant derivative associated with $\gamma^{(0)}$ and all indices are lowered and raised using $\gamma^{(0)}$. Those equations, although easier than the full set of Einstein-Maxwell equations, are still being studied, especially in

\footnotetext{
${ }^{1}$ Einstein equations implies that $\mathcal{F}_{v a}$ pull-backed to the horizon vanishes.
} 
4 and 5 dimensions. Nevertheless, significant results were achieved. For example, in four dimensions they allowed to classify all possible topologies of the horizons in the vacuum case [10], also all axially-symmetric solutions were found [11]. In higher dimensions (first considered in [12]) much less is known because those equations are much less restrictive. For a recent review, see [13].

\subsection{Deformations}

In [14] it was proposed to systematically study extremal black holes in the small $\epsilon$ limit and equations for the first order terms were derived. They were generalized to include matter fields in $[15,16]$. They can be obtained from the Einstein-Maxwell equations satisfied by $(g(\epsilon), \mathcal{F}(\epsilon))$ and differentiating them with respect to $\epsilon$. Those equations, although linear, are rather lengthy and not especially illuminating. Their special case is presented below as (2.9) and (2.10). Interested readers should consult original papers on the topic.

One could naively think that those results are valid only in linearized gravity, since they involve expansion in the small parameter. However, these equations also appear as part of the exact constraints on the characteristic Cauchy data defined on a degenerate (extremal) Killing horizon [17], (or, more generally, on an extremal isolated horizon that is Killing to the appropriate order). The knowledge of this connection is necessary to obtain a well-posed characteristic initial value problem. On the other hand, one could truly interpret solutions to (2.9) and (2.10) as zero-modes living on the background of the near-horizon geometry. Thus, they could be of some interest in the holographic context.

The rest of the paper is organized as follows: in section 2 we present the most general deformation and sketch reasoning behind the derivation. We discuss its properties in section 3. Technical details are relegated to the appendix A.

\section{Classification of the transverse deformations}

\subsection{Background}

We consider the following solution to the Einstein-Maxwell equations: ${ }^{2}$

$$
\begin{aligned}
& g=2 d v\left(d r+\frac{1}{2} r^{2} F d v\right)+r_{+}^{2}\left(\frac{d x^{2}}{1-x^{2}}+\left(1-x^{2}\right) d \phi^{2}\right) \\
& \mathcal{F}=\Psi d v \wedge d r
\end{aligned}
$$

where $\Psi=-\frac{Q}{r_{+}^{2}}, Q$ is an electric charge, $F=\Lambda-\Psi^{2}$ and $r_{+}$is an area radius of the horizon. They satisfy

$$
\frac{1}{r_{+}^{2}}=\Lambda+\Psi^{2}
$$

and so

$$
r_{+}=\sqrt{\frac{2 Q^{2}}{1 \pm \sqrt{1-4 Q^{2} \Lambda}}} .
$$

\footnotetext{
${ }^{2}$ Notice that for simplicity we changed notation with respect to the previous section.
} 
Solution (2.1) can be obtained as a near-horizon limit of the extremal Reissner-Nordström(A)dS black hole. It will serve a rôle of the background for the horizon deformations (which can be seen as well as a zero-modes in this spacetime). For future convenience, let us denote metric induced on the surfaces $r, v=$ const. as $q=r_{+}^{2} \stackrel{q}{q}$. We will denote geometrical objects associated with $\stackrel{q}{q}$ by a small circle.

A few remarks are in place:

(i) It is clear that when $\Lambda>0$, there is an upper bound on the charge. It should not come as a surprise, since the mass is bounded as well.

(ii) When $\Lambda>0$, for the given charge, there are two possible values of $r_{+}$. Indeed, generically, a Reissner-Nordstrom-dS black hole posses three horizons. Extremal case corresponds to the merger of either two of those.

(iii) Solution (2.1) is not the most general spherically symmetric near horizon geometry in the Einstein-Maxwell theory, since one could employ magnetic field as well. The general case reads:

$$
\begin{aligned}
g & =2 d v\left(d r+r^{2}\left(\Lambda-\Psi^{2}-\frac{1}{2} B_{a b} B^{a b} d v\right)\right)+r_{+}^{2}\left(\frac{d x^{2}}{1-x^{2}}+\left(1-x^{2}\right) d \phi^{2}\right) \\
\mathcal{F} & =\Psi d v \wedge d r+\frac{1}{2} B_{a b} d x^{a} \wedge d x^{b}
\end{aligned}
$$

where $B_{a b}=Q_{m} \stackrel{\circ}{\epsilon}_{a b}$ and the radius is given by

$$
\frac{1}{r_{+}^{2}}=\Lambda+\Psi^{2}+\frac{1}{2} B_{a b} B^{a b}
$$

However, the latter can be obtained from the former by a simple dual symmetry:

$$
\begin{aligned}
Q & \mapsto \sqrt{Q_{e}^{2}+Q_{m}^{2}} \\
\mathcal{F} & \mapsto \frac{q_{e}}{\sqrt{Q_{e}^{2}+Q_{m}^{2}}} \mathcal{F}-\frac{Q_{m}}{\sqrt{Q_{e}^{2}+Q_{m}^{2}}} \star \mathcal{F},
\end{aligned}
$$

where $\star$ is a Hodge dual associated with $g$. Since this field rotation is a symmetry of Einstein-Maxwell theory, we can assume $B_{a b}=0$ without loss of generality. Indeed, one can simply apply dual transformation to the deformed solution, which would transform background and deformation separately.

(iv) In higher dimensions, the aforementioned magnetic term breaks the spherical symmetry, since there is no spherically symmetric two-form $B_{a b}$ on $\mathbb{S}^{d}$ when $d>2$. Thus, our choice shall allow for a simpler generalization to the higher-dimensional horizons. We will discuss in more details in the section 3.

\subsection{Deformations}

We can now consider transverse deformations of (2.1). Our discussion will follow closely the derivation of [16] with a small changes in notation. At the leading order, the general 
form of the deformation is

$$
\begin{aligned}
& \delta g=r^{3} F^{(1)} d v^{2}+2 r^{2} h_{a}^{(1)} d v d x^{a}+r \gamma_{a b}^{(1)} d x^{a} d x^{b} \\
& \delta \mathcal{F}=r \Psi^{(1)} d v \wedge d r+r^{2} W_{a}^{(1)} d v \wedge d x^{a}+Z_{a}^{(1)} d r \wedge d x^{a}+\frac{1}{2} r B_{a b}^{(1)} d x^{a} \wedge d x^{b} .
\end{aligned}
$$

All terms written above are $r$ and $v$ independent. Data $\left(F^{(1)}, h_{a}^{(1)}, \Psi^{(1)}, W_{a}^{(1)}, B_{a b}^{(1)}\right)$ can be obtained algebraically from $\gamma_{a b}^{(1)}, Z_{a}^{(1)}$ as follows:

$$
\begin{aligned}
h_{a}^{(1)} & =-\frac{1}{2} D^{b} \gamma_{a b}+\frac{1}{2} D_{a} \gamma+2 \Psi Z_{a}^{(1)} \\
F^{(1)} & =-\frac{1}{3} D^{a} h_{a}^{(1)}-\frac{1}{6}\left(\Lambda-\Psi^{2}\right) \gamma-\frac{2}{3} \Psi \Psi^{(1)} \\
\Psi^{(1)} & =D^{a} Z_{a}-\frac{1}{2} \Psi \gamma^{(1)} \\
W^{(1)} & =\frac{1}{2} d \Psi^{(1)} \\
B^{(1)} & =d Z^{(1)}
\end{aligned}
$$

where $\gamma^{(1)}=q^{a b} \gamma_{a b}^{(1)}$. From the Einstein equations, the remaining equation for $\gamma_{a b}^{(1)}$ follows ${ }^{3}$

$$
0=\Delta_{L} \gamma_{a b}^{(1)}+\frac{1}{2} D_{(a} D_{b)} \gamma^{(1)}-2 \Psi^{2}\left(\gamma_{a b}^{(1)}-\frac{1}{2} \gamma^{(1)} q_{a b}\right)+4 \Psi\left(D_{(a} Z_{b)}-\frac{1}{2} D^{c} Z_{c} q_{a b}\right),
$$

where $\Delta_{L} \gamma_{a b}^{(1)}=-\frac{1}{2} \Delta \gamma_{a b}+R\left(\gamma_{a b}^{(1)}-\frac{1}{2} \gamma^{(1)} q_{a b}\right)$ is a Lichnerowicz operator associated with $q_{a b}$ and $\Delta=q^{a b} D_{a} D_{b}$. Notice that these equations are automatically traceless. Maxwell equations on $Z_{a}$ on the other hand read:

$$
0=\Delta Z_{a}^{(1)}-\frac{1}{r_{+}^{2}} Z_{a}^{(1)}-4 \Psi^{2} Z_{a}^{(1)}-2 F Z_{a}^{(1)}+\Psi D^{b} \gamma_{a b}^{(1)}-\frac{3}{2} \Psi D_{a} \gamma^{(1)}
$$

Our theory can be regarded as a linearized gravity, and thus it enjoys a large group of gauge transformations:

$$
\begin{gathered}
\delta g \mapsto \delta g+\mathcal{L}_{\xi} g \\
\delta \mathcal{F} \mapsto \delta \mathcal{F}+\mathcal{L}_{\xi} \mathcal{F} .
\end{gathered}
$$

Since we consider a very specific form of $\delta g, \delta \mathcal{F}$, vector fields $\xi$ generating gauge transformations must preserve it. It is quite a severe restriction, the most general allowed $\xi$ (modulo isometries of $g$ ) is:

$$
\xi=-\frac{1}{2} f \partial_{v}+\frac{1}{2} r D^{a} f \partial_{a},
$$

where $f=f\left(x^{a}\right)$ is an arbitrary smooth function on a sphere. Geometrically, gauge symmetry is equivalent to the freedom in choosing a cross-section $v=0$ of the horizon. Action

\footnotetext{
${ }^{3}$ Notice that those equations are already evaluated at the background of (2.1) and thus are much simpler.
} 
of $\xi$ can be explicitly written as

$$
\begin{aligned}
\gamma_{a b}^{(1)} & \mapsto \gamma_{a b}^{(1)}+D_{a} D_{b} f \\
h_{a}^{(1)} & \mapsto h_{a}^{(1)}-\frac{1}{2} F D_{a} f \\
F^{(1)} & \mapsto F^{(1)}+\frac{1}{2} D^{a} f D_{a} F \\
\Psi^{(1)} & \mapsto \Psi^{(1)} \\
Z_{a}^{(1)} & \mapsto Z_{a}^{(1)}+\frac{1}{2} \Psi D_{a} f .
\end{aligned}
$$

It is easy to check that the equations of motion are invariant with respect to those transformations. Thus, it may be concluded that eq. (2.9) and (2.10) are undetermined. However, it was shown in [15] that they become elliptic once properly gauged (see [14] for a proof in the absence of an electromagnetic field). It follows from the Fredholm theory that the moduli of solutions (up to the gauge transformations) is finite dimensional. One globally available gauge condition is $\gamma^{(1)}=$ const. However, we will not impose it but rather work with gauge invariant potentials for $\left(\gamma_{a b}^{(1)}, Z_{a}^{(1)}\right)$ from the start.

\subsection{Solutions}

In this section we will write down the solutions to eq. (2.9) and (2.10) and discuss when they exist. Derivation, which is quite lengthy, is redirected to the appendix A.

The most general solution to the eq. (2.10) is:

$$
\begin{aligned}
\gamma_{a b}^{(1)} & =-2\left(\stackrel{\circ}{\epsilon}_{c(a} \stackrel{\circ}{D}_{b)} \stackrel{\circ}{D}^{c}\right)(\stackrel{\circ}{\Delta}-2) \chi+\left(\stackrel{\circ}{D}_{a} \stackrel{\circ}{D}_{b}-\frac{1}{4} q_{a b}(\stackrel{\circ}{\Delta}-2)\right) \Phi+\stackrel{\circ}{D}_{a} \stackrel{\circ}{D}_{b} f \\
Z_{a} & =-\Psi \epsilon_{a c} \stackrel{\circ}{ }^{c}(\stackrel{\circ}{\Delta}+2) \chi+\frac{1}{2} \Psi \stackrel{D}{D}_{a} f,
\end{aligned}
$$

where $\stackrel{\circ}{D}$ is a covariant derivative associated with $\stackrel{q}{q}, \stackrel{\circ}{\Delta}=\stackrel{q}{q}^{a b} \stackrel{\circ}{D}_{a} \stackrel{\circ}{D}_{b}$ and $\chi$ does not have $l=1$ components in the decomposition on the spherical harmonics, ${ }^{4}$ it means

$$
\int_{S^{2}} Y_{1 m}^{\star} \chi=0
$$

Moreover, notice that $l=1$ component of $\Phi$ and $l=0$ component of $\chi$ do not affect $\left(\gamma_{a b}^{(1)}, Z_{a}^{(1)}\right)$ and thus we will set them to zero for simplicity. Inserting those solutions to $(2.9)$ leads to

$$
\begin{aligned}
\left(\stackrel{\triangleright}{\Delta}-2+8 r_{+}^{2} \Psi^{2}\right) \Phi & =\text { const. } \\
\stackrel{\circ}{\Delta}\left(\stackrel{\circ}{\Delta}-2+8 r_{+}^{2} \Psi^{2}\right) \chi & =0
\end{aligned}
$$

Thus, there is always a $\Phi=$ const. solution. For generic $Q, \Lambda$ operator $\left(\stackrel{\diamond}{\Delta}-2+8 r_{+}^{2} \Psi^{2}\right)$ is invertible, and thus it is the only solution. It is not invertible only when

$$
4 Q^{2} \Lambda=1-\left[\frac{l(l+1)-2}{4}\right]^{2}
$$

\footnotetext{
${ }^{4}$ From now by $l$ - components we will mean components in the aforementioned decomposition.
} 
for $l \geq 2$ in which case $\chi$ and $\phi$ can be arbitrary linear combinations of spherical harmonics with given $l$ number. ${ }^{5}$ Notice that when $l=2$, one obtains $\Lambda=0$ and $\Lambda<0$ whenever $l$ is larger.

Thus, we can formulate our main result:

Theorem 1. All solutions to the system of equations (2.9) and (2.10) are of the form:

$$
\begin{aligned}
\gamma_{a b}^{(1)} & =-2\left(\stackrel{\circ}{\epsilon}_{c(a} \stackrel{\circ}{D}_{b)} \stackrel{\circ}{D}^{c}\right)(\stackrel{\circ}{\Delta}-2) \chi+\left(\stackrel{\circ}{D}_{a} \stackrel{\circ}{D}_{b}-\frac{1}{4} q_{a b}(\stackrel{\circ}{\Delta}-2)\right) \Phi+\stackrel{\circ}{D}_{a} \stackrel{\circ}{D}_{b} f \\
Z_{a} & =-\Psi \epsilon_{a c} \stackrel{\circ}{D}^{c}(\stackrel{\circ}{\Delta}+2) \chi+\frac{1}{2} \Psi \stackrel{\circ}{D}_{a} f,
\end{aligned}
$$

where

- $\chi=0, \Phi=c$ when $\Lambda>0$

- $\chi=\sum_{m=-2}^{2} a_{2 m} Y_{2 m}, \Phi=c+\sum_{m=-2}^{2} b_{2 m} Y_{2 m}$ when $\Lambda=0$

- $\chi=\sum_{m=-l}^{l} a_{l m} Y_{l m}, \Phi=c+\sum_{m=-l}^{l} b_{l m} Y_{l m}$ when $4 Q^{2} \Lambda=1-\left[\frac{l(l+1)-2}{4}\right]^{2}$ for $l \in \mathbb{N}_{>2}$

- $\chi=0, \Phi=c$ when $\Lambda<0$ and not included above,

where $a_{l m}, b_{l m}, c$ are constants and $f$ is an arbitrary function which does not encode any physical degrees of freedom.

Moreover, a necessary condition for the horizon to be still a marginally outer trapped surface (MOTS) after deformation is [14]

$$
\int_{S^{2}} \gamma^{(1)}>0
$$

which implies $c>0$.

\section{Discussion}

\subsection{Physical interpretation}

Stationary spacetime is static when its time-like Killing vector is hypersurface orthogonal, it means

$$
K_{[a} \nabla_{b} K_{c]}=0
$$

In the case of linear deformations on the static background, ${ }^{6}$ it reads (up to the first order in $\epsilon$ )

$$
\begin{aligned}
d h^{(1)} & =0 \\
d F^{(1)} & =0 .
\end{aligned}
$$

\footnotetext{
${ }^{5}$ Notice that we excluded $l=1$ case earlier on and $l=0$ does not contribute anything new.

${ }^{6}$ In fact, it may be shown that (2.4) is the most general near horizon geometry for a static, extremal Killing horizon in Einstein-Maxwell theory with compact cross-sections [9].
} 
It can be checked that this is equivalent to $\chi=0$. Thus, when non-trivial deformations exists, we still can have static spacetimes. It would be of interest to find such solutions. For simplicity, one could restrict themselves to the axially symmetric case (it means that $\Phi$ is a linear combination of a constant and of $Y_{l, m=0}$ spherical harmonic). Unfortunately, when $\Lambda \neq 0$ standard techniques of finding static, axially symmetric solutions do not work and so it is highly non-trivial task. On the other hand, it could be reasonably simpler to find them numerically around the horizon.

So far we were mainly focused on the non-generic case. Perhaps more interesting case is the generic one in which only the spherically symmetric deformations exist. It suggests (although does not prove!) that certain spacetimes (e.g. Ernst ones) cannot be generalized to the case of non-vanishing cosmological constant. In particular, a stationary embedding of the electrically charged Reissner-Nordström black hole into the magnetic field ${ }^{7}$ seems to be excluded. Of course, it may happen that such spacetimes exist, but their leading-order deformations are just spherically symmetric. Nevertheless, it is a noticeable difference in contrast to the $\Lambda=0$ case.

In the asymptotically flat setting, one could also have Majumdar-Papapetrou spacetime, which describes multi-centered extremal black holes in which gravity and electromagnetism balance each other. As checked in [16], their leading-order deformation is exactly spherically symmetric, so our result cannot tell anything about the existence of such black holes. ${ }^{8}$

It seems from this discussion that it is worth investigating is going beyond the first order perturbation in $\epsilon$. The extremely simple form of the solutions in the first order (at least, for generic values of charge) encourages one to go beyond that. If the spherical symmetry persisted, it would be a rather strong argument for the uniqueness of the Reissner-Nordström-(A)dS spacetimes. We plan to address it in the future work.

\subsection{Connection to the previous work}

As already mentioned, the systematic study on the extremal horizons' transverse deformations was initiated in [14] with further developments in [15-17]. In particular, in [16] all axially symmetric deformations, which consists a 3-dimensional family (including spherically symmetric one), of Reissner-Nordström were found.

We were able to remove this symmetry assumption in our work, discovering 11dimensional space of allowed deformations. Obviously, since eq. (2.9) and (2.10) are linear, one can superpose solutions which are axially symmetric with respect to the different choices of axis. (Whether they are realized as a solution to the non-linear Einstein-Maxwell equations is an entirely different matter, which lies beyond the scope of this work.) However, they span only 7-dimensional space, and thus they do not cover all solutions presented here. Unfortunately, we are not aware of any extremal black holes solutions without spatial symmetry, so we do not know whether those linearized deformations can be embedded into

\footnotetext{
${ }^{7}$ Note that generic Reissner-Nordström-Melvin suffer from the conical singularity and thus are excluded since we are working with smooth objects from the first.

${ }^{8}$ In fact, multi-centered black hole solutions with $\Lambda>0$ are known [18]. However, they do not possess any Killing vector and thus are excluded from our analysis.
} 
the non-linear solution. Of course, from the rigidity theorem, it follows that such solutions could not be asymptotically flat. Axially-symmetric case is much better understood and large portion of the solutions space was embedded (into Ernst solutions and very special cases of Kerr-Melvin ones).

The problem of finding solutions to (2.9) and (2.10) with non-zero cosmological constant and charge were not investigated so far in the literature. ${ }^{9}$ Since for generic charge we find only spherically symmetric deformations, there is no problem with an embedding into well-known solutions. Much more interesting are backgrounds with non-generic charges, since they admit a plethora of deformations. Unfortunately, we are not aware of any exact solutions to the Einstein-Maxwell equations in which such particular values of charges were distinguished, so for now their meaning is rather obscure. Even if they exist, they seem to be highly non-generic, even for the standards of the extremal black holes, in vast contrast with the $\Lambda=0$ case. Perhaps this work could serve as a suggestion that this fine-tuning is needed for constructing new, interesting solutions. It may be possible to construct such spacetimes numerically. Unfortunately, all black holes found in [8] have positive Hawking temperature and thus cannot describe what we are looking for. However, certain generalization of this work is possible, namely by introducing $l=0$ mode in the boundary conditions at infinity. Since horizons considered in this work are charged, we conjecture that inclusion of this mode with a fine-tuned boundary conditions would yield extremal, non-trivial black holes.

\subsection{Generalizations and further work}

\subsubsection{Higher dimensional horizons}

For now we restricted ourselves only to the four-dimensional spacetimes. Nevertheless, equations for the deformations in the Einstein-Maxwell theory were derived in an arbitrary dimension [16]. It can be easily seen that

$$
\tilde{\gamma}_{a b}=\gamma_{a b}+\frac{2}{\Psi} D_{(a} Z_{b)}
$$

is still a gauge invariant quantity. ${ }^{10}$ Hodge decomposition on $S^{d}$ reads

$$
Z_{a}=D^{b} \omega_{a b}+D_{a} f
$$

where $\omega_{a b}$ is a gauge-invariant two-form (defined up to the term which is $\star d \star$-exact). It seems that one could rewrite all equations using those invariant objects and hopefully solve them again due to the simplicity offered by a spherical symmetry. We hope to address it in the future.

Moreover, in higher-dimensional theories different matter fields could be included (e.g. Chern-Simons term in odd-dimensional ones), those were described in detail in [15]. Of

\footnotetext{
${ }^{9}$ Having finished this work, we were informed by James Lucietti about his unpublished result regarding classification of the axially-symmetric deformations of the Reissner-Nordström-(A)dS black holes which agrees with the solutions presented here.

${ }^{10}$ It would be still true if one omitted symmetrization in $D_{a} Z_{b}$. Such non-symmetric $\tilde{\gamma}_{a b}$ may be proven to be more useful.
} 
course, one should start by investigating the simplest possible horizons, it means those which cross-sections are maximally-symmetric. In particular, in $d=5$, all homogenous horizons in the Einstein-Maxwell-Chern-Simons theory were classified in [13].

\subsubsection{Beyond spherical symmetry}

Our arguments so far depend heavily upon the assumption of the spherical symmetry of the background. Obviously, the most interesting background would be Kerr-Newman(A)dS which is only axially symmetric. Unfortunately, we were not able to construct gauge invariant quantities like $\tilde{\gamma}_{a b}$ above. Instead of looking for them, one could split $\gamma_{a b}, Z_{a}$ into Fourier modes with respect to the Killing vector field $\partial_{\phi}$. This reduces the problem to the system of (undetermined, due to the gauge symmetry) ODEs. ${ }^{11}$ Unfortunately, solving such a system is again a non-trivial task. In the background of both Kerr and Kerr-AdS it was shown that the only axially symmetric solutions are gauge equivalent to the Kerr and Kerr-AdS itself, respectively. It would be of interest from the point of view of both AdS/CFT correspondence and uniqueness of black holes to determine whether this is still true without additional symmetry assumptions.

\section{Acknowledgments}

It is a pleasure to thank Eryk Buk, Jerzy Lewandowski and James Lucietti for useful discussions. This work was financed from budgetary funds for science in 2018-2022 as a research project under the program "Diamentowy Grant".

\section{A Technical details}

In this section we will describe in more details how Theorem 1 can be derived. For simplicity, we will omit subscript (1).

Let us start by a simple observation that a quantity

$$
\tilde{\gamma}_{a b}:=\gamma_{a b}-\frac{2}{\Psi} D_{(a} Z_{b)}
$$

is gauge invariant. Moreover, we have Hodge decomposition of $Z_{a}$ :

$$
Z_{a}=\Psi \epsilon_{a c} D^{c} S+\Psi D_{a} f
$$

in which $f$ is pure gauge and $S$ is gauge-invariant. (Since $\Psi$ is a non-vanishing constant, it can multiply both terms without loss of generality.) For simplicity, we will multiply both eq. (2.9) and (2.10) by $r_{+}^{2}$. In this way we describe everything in terms of geometrical objects associated with $\stackrel{\leftrightarrow}{q}_{a b}$. Inserting $\tilde{\gamma}_{a b}$ to $(2.10)$ leads to

$$
\Psi \stackrel{\circ}{D}^{b}\left(\tilde{\gamma}_{a b}-\frac{3}{2} \tilde{\gamma} \stackrel{\circ}{a b}\right)=-2\left(\stackrel{\circ}{\Delta} \delta_{c}^{b}-\delta_{c}^{b}-\stackrel{\circ}{D}_{a} \stackrel{\circ}{D}^{b}\right) Z_{b},
$$

where $\tilde{\gamma}=q^{a b} \tilde{\gamma}_{a b}$. Since the left-hand side is gauge-invariant, so must be the right-hand one. Indeed, it is equal to $-2 \Psi \epsilon_{a b} D^{b} \stackrel{\circ}{\Delta}$. Any $\tilde{\gamma}_{a b}-\frac{3}{2} \tilde{\gamma} q_{a b}$ satisfying this equation can be uniquely

\footnotetext{
${ }^{11}$ Indeed, investigation of the axially symmetric deformations of the Kerr-AdS in [16] relies exactly on this.
} 
decomposed into the trace-free and divergence-free part, since there are no non-vanishing trace-free, divergence-free symmetric tensors on $S^{2}$. The most general divergence-free tensor can be written as:

$$
s_{a b}=\left(\stackrel{\circ}{D}_{a} \stackrel{\circ}{D}_{b}-(\stackrel{\circ}{\Delta}+1) \stackrel{\circ}{q}_{a b}\right) \Phi .
$$

Notice that $\stackrel{a}{q}^{a b} s_{a b}$ is an arbitrary function with an exception that it does not possess $l=1$ components, which follows from the following

Lemma 1. Let $s_{a b}=s_{(a b)}, \stackrel{\circ}{D}^{a} s_{a b}=0$. Then

$$
\int_{S^{2}} Y_{1 m}^{\star} \stackrel{\stackrel{q}{ }}{a b}^{a b}=0
$$

We will prove this lemma by contradiction. We already constructed solutions with an arbitrary trace without $l=1$ components, so we can assume without loss of generality that $\stackrel{\mathrm{q}}{q}^{a b} s_{a b}=: f$ has only a dipole part. Then, $D^{b} f$ is a conformal Killing vector field on $S^{2}$ and

$$
\stackrel{\circ}{D}_{a} \stackrel{\circ}{D}_{b} f=\frac{1}{2} \stackrel{\circ}{\Delta} f \stackrel{\circ}{q}_{a b}
$$

Let us calculate:

$$
\stackrel{\circ}{D}^{a}\left(s_{a b} \stackrel{\circ}{D}^{b} f\right)=s_{a b} \stackrel{\circ}{D}^{a} \stackrel{\circ}{D}^{b} f=\frac{1}{2} s \stackrel{\circ}{\Delta} s .
$$

Integrating this expression on $S^{2}$ we obtain

$$
0=\int_{S^{2}} \stackrel{\circ}{D}^{a}\left(s_{a b} \stackrel{\circ}{D}^{b} f\right)=\int_{S^{2}} \frac{1}{2} s \stackrel{\circ}{\Delta} s=-\frac{1}{2} \int_{S^{2}} \stackrel{\circ}{D}^{a} s \stackrel{\circ}{D}_{a} s
$$

Thus we conclude that $s=$ const. $=0$. This proves this lemma and shows that (A.4) is the most general divergence-free symmetric 2-expression. We are thus left with a task of finding any solution to the non-homogenous problem. Natural ansatz is

$$
s_{a b}=2 \stackrel{\circ}{D}_{(a} \epsilon_{b) c} U
$$

which leads to

$$
\epsilon_{b c} \stackrel{\circ}{D}^{c}(\stackrel{\circ}{\Delta}+2) U=-2 \epsilon_{b c} \stackrel{\circ}{D}^{c} \Delta S,
$$

which can be solved by

$$
\begin{aligned}
& U=2 \stackrel{\circ}{\Delta} \\
& S=-(\stackrel{\circ}{\Delta}+2) \chi
\end{aligned}
$$

unless $S$ has a non-vanishing dipole part. This case can be excluded in the way very similar to the Lemma 1. Indeed, without loss of generality, we may assume that $S$ has only dipole part. Then, $-2 \epsilon_{b c} \stackrel{\circ}{ }^{c} \Delta S=: K_{b}$ is a Killing covector field and let $\stackrel{\circ}{D}^{a} s_{a b}=K_{b}$. One can easily calculate:

$$
\stackrel{\circ}{D}^{a}\left(s_{a b} K^{b}\right)=K^{2}
$$


Integrating both sides, we get $K^{2}=0$. Thus, the most general solution to $(2.10)$ is

$$
\begin{aligned}
\tilde{\gamma}_{a b}-\frac{3}{2} \tilde{\gamma} q_{a b} & =-4 \stackrel{\circ}{\epsilon}_{c(b} \stackrel{\circ}{D}_{a)} \stackrel{\circ}{ }^{c} \stackrel{\circ}{\Delta} \chi+\left(\stackrel{\circ}{D}_{a} \stackrel{\circ}{D}_{b}-\stackrel{\circ}{q}_{a b}(\stackrel{\circ}{\Delta}+1)\right) \Phi \\
Z_{a} & =-\Psi \epsilon_{a b} \stackrel{\circ}{D}^{b}(\stackrel{\circ}{\Delta}+2) \chi .
\end{aligned}
$$

After an easy algebra, one gets (2.14). Inserting it to (2.9) leads to

$$
\left(\stackrel{\circ}{D}_{a} \stackrel{\circ}{D}_{b}-\frac{1}{2} q_{a b} \stackrel{\circ}{\Delta}\right) W(\Phi)+\left(\stackrel{\circ}{\epsilon}_{c(b} \stackrel{\circ}{D}_{a)} \stackrel{\circ}{D}^{c}\right) V(\chi)=0
$$

where

$$
\begin{aligned}
W(\Phi) & =\left(-\frac{1}{4} \stackrel{\circ}{\Delta}+\frac{1}{2}-2 r_{+}^{2} \Psi^{2}\right) \Phi \\
V(\chi) & =\stackrel{\circ}{\Delta}\left(\stackrel{\Delta}{\Delta}-2+8 r_{+}^{2} \Psi^{2}\right) \chi
\end{aligned}
$$

Taking two divergences of (A.14) leads to

$$
0=\frac{1}{2} \stackrel{\circ}{\Delta}(\stackrel{\circ}{\Delta}+2) W
$$

which holds only when $W$ is a linear combination of $l=0$ and $l=1$ spherical harmonics and then

$$
\left(\stackrel{\circ}{D}_{a} \stackrel{\circ}{D}_{b}-\frac{1}{2} \stackrel{\circ}{q}_{a b} \stackrel{\circ}{\Delta}\right) W=0
$$

is automatically satisfied and one is left with

$$
\left(\stackrel{\circ}{\epsilon}_{c(b} \stackrel{\circ}{D}_{a)} \stackrel{\circ}{D}^{c}\right) V=0
$$

Multiplying by $\stackrel{\circ}{\epsilon}_{d}^{b}$ leads to

$$
2\left(\stackrel{\circ}{D}_{a} \stackrel{\circ}{D}_{d}-\frac{1}{2} \stackrel{\circ}{q}_{a d} \stackrel{\circ}{\Delta}\right) V=0
$$

and so also $V$ is a linear combination of $l=0$ and $l=1$ spherical harmonics. However, we already deduced that $\chi$ cannot have $l=1$ components and its $l=0$ component was set to zero and thus $V=0$. Also $l=1$ part of $\Phi$ is meaningless and thus $W=$ const. which leads to $(2.16)$.

Open Access. This article is distributed under the terms of the Creative Commons Attribution License (CC-BY 4.0), which permits any use, distribution and reproduction in any medium, provided the original author(s) and source are credited.

\section{References}

[1] W. Israel, Event horizons in static vacuum space-times, Phys. Rev. 164 (1967) 1776 [INSPIRE].

[2] S.W. Hawking, Black holes in general relativity, Commun. Math. Phys. 25 (1972) 152 [INSPIRE]. 
[3] B. Carter, Axisymmetric black hole has only two degrees of freedom, Phys. Rev. Lett. 26 (1971) 331 [INSPIRE].

[4] D.C. Robinson, Uniqueness of the Kerr black hole, Phys. Rev. Lett. 34 (1975) 905 [inSPIRE].

[5] P.O. Mazur, Proof of uniqueness of the Kerr-Newman black hole solution, J. Phys. A 15 (1982) 3173 [INSPIRE].

[6] P.T. Chrusciel and R.M. Wald, On the topology of stationary black holes, Class. Quant. Grav. 11 (1994) L147 [gr-qc/9410004] [INSPIRE].

[7] T.M. Adamo, C.N. Kozameh and E.T. Newman, Null geodesic congruences, asymptotically flat space-times and their physical interpretation, Living Rev. Rel. 12 (2009) 6 [arXiv:0906.2155] [INSPIRE].

[8] C.A.R. Herdeiro and E. Radu, Static Einstein-Maxwell black holes with no spatial isometries in AdS space, Phys. Rev. Lett. 117 (2016) 221102 [arXiv:1606.02302] [INSPIRE].

[9] H.K. Kunduri and J. Lucietti, Uniqueness of near-horizon geometries of rotating extremal $A d S_{4}$ black holes, Class. Quant. Grav. 26 (2009) 055019 [arXiv:0812.1576] [InSPIRE].

[10] D. Dobkowski-Rylko, W. Kamiński, J. Lewandowski and A. Szereszewski, The Near Horizon Geometry equation on compact 2-manifolds including the general solution for $g>0$, Phys. Lett. B 785 (2018) 381 [arXiv: 1807.05934] [INSPIRE].

[11] J. Lewandowski and T. Pawlowski, Extremal isolated horizons: a Local uniqueness theorem, Class. Quant. Grav. 20 (2003) 587 [gr-qc/0208032] [INSPIRE].

[12] J. Lewandowski and T. Pawlowski, Quasi-local rotating black holes in higher dimension: geometry, Class. Quant. Grav. 22 (2005) 1573 [gr-qc/0410146] [INSPIRE].

[13] H.K. Kunduri and J. Lucietti, Classification of near-horizon geometries of extremal black holes, Living Rev. Rel. 16 (2013) 8 [arXiv:1306.2517] [INSPIRE].

[14] C. Li and J. Lucietti, Transverse deformations of extreme horizons, Class. Quant. Grav. 33 (2016) 075015 [arXiv: 1509.03469] [INSPIRE].

[15] A. Fontanella and J.B. Gutowski, Moduli spaces of transverse deformations of near-horizon geometries, J. Phys. A 50 (2017) 215202 [arXiv: 1610.09949] [InSPIRE].

[16] C. Li and J. Lucietti, Electrovacuum spacetime near an extreme horizon, Adv. Theor. Math. Phys. 23 (2019) 1903 [arXiv: 1809.08164] [INSPIRE].

[17] M. Kolanowski, J. Lewandowski and A. Szereszewski, Extremal horizons stationary to the second order: new constraints, Phys. Rev. D 100 (2019) 104057 [arXiv:1907.00955] [INSPIRE].

[18] D. Kastor and J.H. Traschen, Cosmological multi-black hole solutions, Phys. Rev. D 47 (1993) 5370 [hep-th/9212035] [INSPIRE]. 